\title{
Educación matemática inclusiva: posibilidades y acercamientos a un programa de maestría en Boyacá (Colombia)
}

\author{
José W. González ${ }^{1 *}$, Alexandra González ${ }^{2}$, y José E. Cifuentes ${ }^{1}$ \\ (1) Universidad Pedagógica y Tecnológica de Colombia, Facultad de Estudios a Distancia, Lic. en Educación Básica \\ Primaria, Tunja, Colombia. (correo-e: joseweymar.gonzalez@uptc.edu.co; joseeriberto.cifuentes@uptc.edu.co) \\ (2) Universidad Pedagógica y Tecnológica de Colombia, Facultad de Estudios a Distancia, Esc. de Ciencias Tecnológicas, \\ Tunja, Colombia. (correo-e: alexandra.gonzalez@uptc.edu.co)
}

* Autor a quien debe ser dirigida la correspondencia

Recibido Sep. 22, 2020; Aceptado Nov. 16, 2020; Versión final Ene. 12, 2021, Publicado Abr. 2021

\begin{abstract}
Resumen
Este trabajo tiene como objetivo determinar las necesidades de formación en educación matemática inclusiva a nivel de maestría en el departamento de Boyacá (Colombia), mediante la indagación de la oferta y demanda de dicho programa y las oportunidades de desempeño para el egresado. Se utiliza un enfoque de investigación mixto de tipo exploratorio, se aplican cuestionarios a docentes de matemáticas, entrevistas a expertos de educación y se realiza una revisión documental. Se encuentra que no existe oferta del programa de maestría o similares en la región. Los docentes tienen dificultades para enseñar en contextos de discapacidad y diversidad debido a su bajo nivel de cualificación en educación inclusiva, pero existen oportunidades de desempeño para el egresado. Se concluye que existen necesidades de formación en educación matemática inclusiva en los docentes del departamento de Boyacá y poca oferta educativa que las supla, lo cual representa una oportunidad para diseñar un programa de maestría en Boyacá.
\end{abstract}

Palabras clave: educación inclusiva; matemáticas; posgrado; formación; docentes; discapacidad

\section{Inclusive mathematics education: possibilities and approaches to a master's program in Boyacá (Colombia)}

The aim of this study is to determine the training needs in inclusive mathematics education at the master's level in the department of Boyacá (Colombia) by examining supply and demand and the performance of prospective graduates. A mixed exploratory research approach is used. Questionnaires are applied to mathematics teachers, education experts are interviewed, and a literature review is performed. The results show that there is no offer of a master's program in inclusive mathematics education in the region of Boyacá. Teachers have difficulties teaching in contexts of disability and diversity due to their low level of qualification in inclusive education. However, there are good performance prospects for future graduates. It is concluded that there are training needs in inclusive mathematics education in the department of Boyacá, but there is a lack of educational offers to meet them, opening the opportunity to design a master's program in Boyacá.

Keywords: inclusive education; mathematics; postgraduate; teacher training; disability 


\section{INTRODUCCIÓN}

La educación inclusiva busca que la enseñanza sea eficaz para todos en el aula, sin importar su discapacidad o diversidad; no obstante, pese a que los Estados obligan este tipo de educación en sus países, se ha considerado que los maestros no se encuentran preparados para proporcionarla (Faragher y Clarke, 2016) y generalmente tienen dudas sobre la capacidad de sus estudiantes con discapacidades (Tan y Padilla, 2019). Esto abre campos de investigación académica y curricular que permitan hacer frente a estas debilidades. El trasfondo histórico de la educación inclusiva refleja un cambio en las ideas respecto a la discapacidad, lo que ha llevado a la transformación de modelos segregadores en el siglo XIX a modelos rehabilitadores de integración escolar en la segunda mitad del siglo XX (Petreñas et al., 2020).

Posteriormente, a partir de Declaración mundial sobre Educación para todos y Marco de acción para satisfacer las necesidades básicas de aprendizaje, realizada en Jomtien Tailandia en 1990, la Declaración de Salamanca y Marco de acción sobre las Necesidades Educativas Especiales, realizada en Salamanca España en 1994, y la Convención sobre los Derechos de las Personas con Discapacidad, realizada en Nueva York en 2006, la adopción del modelo social de la discapacidad en el que se empieza a concebir al sujeto con discapacidad desde su diversidad, las barreras para el aprendizaje y participación que la sociedad le impone y las consecuentes situaciones de vulnerabilidad y dificultades en la interacción y desenvolvimiento que estas le ocasionan en la escuela (Ainscow, 2016; Messiou et al., 2016). Desde el modelo social, el problema de aprendizaje no es ocasionado por la diversidad funcional individual del estudiante, sino por las limitaciones de la sociedad en la prestación de servicios básicos y adecuados que aseguren que las personas con discapacidad sean incluidas efectivamente en la organización social.

Dentro de este último modelo, la educación inclusiva es un enfoque que "procura transformar los sistemas educativos y mejorar la calidad de la enseñanza a todos los niveles y en todos los ambientes, con el fin de responder a la diversidad de los educandos y promover un aprendizaje exitoso" (Matsuura, 2008, p. 2), en términos más cortos, es una enseñanza eficaz para todos los alumnos (Ainscow y Miles, 2008). Desde la educación inclusiva, el estudiante con discapacidad no requiere de especialistas que atiendan sus "necesidades educativas especiales", sino de apoyos naturales o ajustes razonables por parte de maestros, padres, amigos y otros cuidadores (Baglieri et al., 2011). Esto significa que es la escuela la que se adapta a las características propias de quienes están "afuera" e implica cambios profundos en los factores físicos, aspectos curriculares, expectativas, estilos de los profesores, roles directivos, entre otros.

Lo anterior es particularmente relevante para la enseñanza matemática, pues las estrategias didácticas se vuelven más complejas y demandan formas eficaces de facilitar el aprendizaje de todos los alumnos cuando en el aula se encuentran estudiantes cuyas diferencias funcionales, de desempeño, cultura, raza, género, etnia, lenguaje, contexto socioeconómico, religión o nacionalidad, inciden negativamente en su desenvolvimiento en la escuela (Ainscow, 2016). Específicamente, estas diferencias pueden limitar el acceso a los contenidos enseñados debido a las dificultades de los estudiantes para ver, escuchar, acceder a materiales, entender el idioma o comprender conceptos y procedimientos matemáticos según sus creencias o contextos socioculturales.

Frente a estos retos, la educación matemática inclusiva se constituye como aquel tipo de educación en el que esta ciencia es accesible y comprensible para todo el mundo, sin renunciar al aprendizaje de conocimientos matemáticos básicos ni llegar a la simplificación del discurso de la enseñanza de las matemáticas (Alsina y Planas, 2010). A nivel internacional las investigaciones alrededor de este tipo de educación han abierto campos de estudio (por ejemplo, los Estudios de Discapacidad en la Educación Matemática, DSME), han reafirmado la discapacidad como una construcción social y se han centrado en ver a los estudiantes con discapacidad como pensadores y practicantes de las matemáticas, en identificar lo que aprenden estos estudiantes y en cómo asegurar el acceso a los contenidos curriculares (Shogren y Wehmeyer, 2014; Tan et al., 2019). Esto demuestra los avances que ha tenido la educación matemática inclusiva como campo de estudio y los retos y las posibilidades que tienen los educadores matemáticos de profesionalizarse por medio de la literatura científica.

Pese a estos progresos, se ha verificado que en la enseñanza de las matemáticas en Latinoamérica existe baja transformación de las prácticas docentes en la escuela a través de programas de formación permanente en educación inclusiva (ALTER-NATIVA, 2013). En efecto, los estudios internacionales han hallado poca preparación de los docentes para suministrar educación matemática inclusiva, dudas que tienen los maestros de la capacidad de los estudiantes con discapacidad y un conjunto reducido de metodologías y pedagogías para su inclusión efectiva (Faragher y Clarke, 2016; Lambert y Tan, 2017; Tan y Padilla, 2019). En Colombia la adopción estatal de la educación inclusiva para todo el sistema educativo en los niveles de básica y media (Decreto 366 de 2009 y Decreto 1421 de 2017) ha planteado nuevos retos para los docentes en sus aulas, quienes deben adaptarse y lograr aprendizajes eficaces en todos sus alumnos. No obstante, el logro de la 
educación inclusiva en Colombia aún está lejos de alcanzarse, pues algunos docentes colombianos indican no sentirse capacitados para educar estudiantes con discapacidad (Hurtado y Agudelo, 2014), esto ha ocasionado que en el país el $56.8 \%$ de las personas con discapacidad, que se encuentran entre los 5 y los 20 años, estén vinculados a procesos de formación básica, pero solo el $5.4 \%$ de ellos logre terminar educación básica y media (Hurtado y Agudelo, 2014).

Específicamente, los docentes del departamento de Boyacá (Colombia) no escapan del reto en educación inclusiva, pues tienen en sus escuelas un total de 3.328 estudiantes caracterizados con discapacidad, 2.659 en condición de víctimas del conflicto armado y 961 pertenecientes a grupos étnicos (Sistema Integrado de Matricula (SIMAT)- Secretaría de Educación de Boyacá, 2019). A pesar de esta necesidad evidente, las acciones de educación inclusiva por parte del gobierno departamental se han enfocado en atención profesional externa, diseño de planes educativos especiales, foros, premiación a experiencias exitosas o la formación docente mediante diplomados, descuidando la profesionalización docente. Por lo tanto, un área que aún permanece inexplorada y, en consecuencia, requiere ser fortalecida, consiste en la cualificación profesional en educación inclusiva a nivel de maestría dirigida a los docentes del departamento, especialmente en matemáticas, una de las áreas de mayor reto académico y priorizada a nivel nacional e internacional para medir la calidad educativa.

Ante estas condiciones sociales, de acuerdo con Florian y Camedda (2020), no disponer de un currículo que promueva las prácticas pedagógicas inclusivas amplía la segregación escolar y disminuye las posibilidades de participación y progreso de todos los estudiantes en el sistema escolar. Asimismo, la existencia de profesores que no estén preparados en educación inclusiva contribuye a la exclusión o perjuicio de personas pertenecientes a algunas minorías en el sistema educativo (Lewin, 2014). Se requiere actuar para evitar estas problemáticas.

De acuerdo a la necesidad planteada, el objetivo de esta investigación es indagar la oferta y demanda de un programa de Educación Matemática Inclusiva a nivel de maestría en el departamento de Boyacá (Colombia), las oportunidades de desempeño para el egresado y la necesidad que se puede vislumbrar en la brecha que existe entre estos componentes. El desarrollo de este objetivo se basa en teorías de diseño curricular por competencias (Santiváñez, 2013), donde la formulación de un programa de formación a nivel de maestría requiere del conocimiento de las condiciones de oferta y demanda del mismo y las condiciones legales para su diseño curricular. A su vez, el enfoque por competencias busca que los estudiantes sean aprendices autónomos, independientes, autorregulados, con capacidad de alcanzar el aprendizaje significativo (Acebedo et al., 2017; León et al., 2014).

\section{METODOLOGÍA}

El enfoque de investigación utilizado es totalizante o mixto, es decir, utiliza de manera combinada métodos cualitativos y cuantitativos con el fin de integrar y discutir los datos recolectados y lograr un mayor entendimiento del fenómeno de estudio (Moscoso, 2017). El diseño de investigación utilizado es exploratorio, que es aplicable para temas poco estudiados; en este caso, un estudio de mercado y de necesidades de un programa de Maestría en Educación Matemática Inclusiva no se ha realizado antes en la región.

Teniendo en cuenta los fundamentos curriculares de Santiváñez (2013) se estructura en esta investigación un modelo de exploración de la oferta y demanda de un programa de formación en Educación Matemática Inclusiva a nivel de maestría. Como se puede observar en la tabla 1, los componentes del modelo son la oferta del programa o similares, la demanda por parte de los docentes y las oportunidades de desempeño que tendría el egresado del programa.

Tabla 1: Componentes del modelo de exploración de la investigación, instrumentos y análisis de información.

\begin{tabular}{|l|l|l|}
\hline Componente del modelo de exploración & Instrumento de recolección & Análisis de datos \\
\hline \multirow{2}{*}{$\begin{array}{l}\text { Oferta del programa o similares en la } \\
\text { región }\end{array}$} & Análisis documental & Tablas documentales de análisis \\
\cline { 2 - 3 } & Entrevista a expertos & Análisis de contenido con Atlas T.I. \\
\cline { 2 - 3 } & Cuestionario a docentes & Análisis estadístico con Excel y SPSS \\
\hline $\begin{array}{l}\text { Demanda del programa en los } \\
\text { docentes de matemáticas }\end{array}$ & Cuestionario a docentes & Análisis estadístico con Excel y SPSS \\
\hline $\begin{array}{l}\text { Oportunidades de desempeño que } \\
\text { tendrían los egresados del programa }\end{array}$ & Análisis documental & Tablas documentales de análisis \\
\cline { 2 - 3 } & Entrevista a expertos & Análisis de contenido con Atlas T.I. \\
\hline
\end{tabular}


Los instrumentos de recolección de la información se construyeron tras un análisis de literatura, elaboración de preguntas según los aspectos de la oferta, demanda y oportunidades de desempeño para los egresados del programa que se querían indagar. Las preguntas se validaron con expertos que tenían postgrado a nivel de doctorado en educación. De acuerdo al enfoque investigativo, se utilizaron distintos instrumentos, para el enfoque cuantitativo y para el enfoque cualitativo.

En el enfoque cuantitativo, se aplicó un cuestionario de opción múltiple a una muestra aleatoria simple de 104 docentes de matemáticas que cumplieron los siguientes criterios de inclusión: pertenecer a uno de los 15 municipios de la provincia Centro del departamento de Boyacá, estar ejerciendo como docente en una institución educativa y ser docente del área de matemáticas. En dicho instrumento se indagó aspectos relativos a las siguientes categorías: realización de estudios respecto a la educación inclusiva, autovaloración de la capacidad de atención de la diversidad, conocimiento de programas académicos sobre educación inclusiva en posgrado e interés en tomar estudios de esta clase. La información se recolectó tras contactar la institución educativa y los docentes y se respondió de manera física en papel y de manera virtual con formulario de Google.

En el enfoque cualitativo, se aplicó una entrevista a cinco expertos en educación inclusiva y diseño curricular de Colombia y España, cuya formación a nivel doctoral y experiencia investigativa está relacionada con matemáticas e inclusión, con el fin de indagar las siguientes categorías de análisis: conocimiento de programas de formación en Educación Matemática Inclusiva a nivel de maestría o similares y campos ocupacionales en los que se pueden desempeñar los egresados del programa. La información se recolectó tras contactar vía correo electrónico a 10 expertos preseleccionados por sus publicaciones respecto a la educación inclusiva y matemáticas. De ellos se obtuvo respuesta de cinco, cuyas opiniones se dieron de manera abierta a las preguntas enviadas.

Además, se realizó una revisión documental en la base de datos del Sistema Nacional de Información de la Educación Superior SNIES de Colombia e internet para conocer la oferta de programas a nivel de posgrado en educación matemática inclusiva. Los criterios de búsqueda fueron: que sea un programa de posgrado en español, que el programa en su denominación mencione la educación inclusiva y/o matemática y que sea un programa vigente. Los datos cuantitativos recolectados se analizaron mediante Excel y su posterior análisis estadístico en el software SPSS para determinar participaciones porcentuales y medidas de tendencia central. Los datos cualitativos se analizaron mediante software Atlas T.I. asignando categorías a la información recolectada y tablas documentales de análisis.

\section{RESULTADOS}

Las condiciones de mercado para un programa de Maestría en Educación Matemática Inclusiva se determinan por medio de la disponibilidad de programas que existen a nivel internacional, nacional y regional que ofrezcan esta área del conocimiento y las necesidades y disposición a profesionalizarse en este programa por parte de los docentes de matemáticas del departamento de Boyacá. A continuación, se desarrollan los hallazgos respecto a la oferta y demanda del programa de maestría en mención y la brecha existente entre estos dos componentes.

\section{Oferta del programa o similares en la región}

Para determinar la oferta de programas de Maestría en Educación Matemática Inclusiva o similares para el departamento de Boyacá (Colombia), debe considerarse la oferta internacional en modalidad virtual y la oferta nacional semipresencial, pues con el avance de la Tecnologías de la Información y las Comunicaciones (TIC) se eliminan las barreras físicas de acceso a la educación posgraduada, lo que obliga a tener en cuenta la oferta más allá de la regional.

En el ámbito internacional existen algunos programas de formación hacia la educación inclusiva en modalidad virtual, pero ninguno de ellos tiene como enfoque de enseñanza explícito la educación matemática inclusiva (ver tabla 2). Pese a ello, esta oferta internacional constituye una posibilidad de formación para los docentes, considerando las opciones de profundización que ellos tienen en su trabajo de grado y las posibilidades de convalidación de dichos programas en Colombia. En Colombia tampoco existen programas de educación matemática inclusiva a nivel de maestría. Sin embargo, la oferta más cercana a dicha formación son los programas de posgrado en el área de educación matemática y programas de posgrado en el área de inclusión social y/o discapacidad. En el primer grupo, se han creado 66 programas de posgrado, pero a febrero de 2020 se encuentran activos 24 de ellos en los siguientes niveles de formación posgraduada: 7 de especialización, 18 de maestría y uno de doctorado (ver tabla 3). 
Tabla 2: Programas de posgrado ofertados en educación inclusiva a nivel internacional, en idioma español y en modalidades virtual y semipresencial.

\begin{tabular}{|l|l|c|c|}
\hline Universidad & Nombre del programa & Modalidad & País \\
\hline $\begin{array}{l}\text { Euroinnova Formación: international } \\
\text { business school. }\end{array}$ & $\begin{array}{l}\text { Máster en Educación, Escuela inclusiva y } \\
\text { atención a la diversidad + titulación universitaria }\end{array}$ & Virtual & España \\
\hline $\begin{array}{l}\text { Instituto de posgrados virtuales } \\
\text { internacionales UENICMLK }\end{array}$ & $\begin{array}{l}\text { Maestría en Educación con Énfasis en } \\
\text { Interculturalidad }\end{array}$ & Nicaragua \\
\hline Universitat de Valéncia & $\begin{array}{l}\text { Máster en atención a la diversidad y educación } \\
\text { inclusiva }\end{array}$ & Virtual & España \\
\hline Universidad de León & $\begin{array}{l}\text { Máster en Educación Inclusiva - Atención a la } \\
\text { diversidad }\end{array}$ & Virtual & México \\
\hline Fundación Extensus & $\begin{array}{l}\text { Maestría en Educación Inclusiva: Formación y } \\
\text { respuesta educativa en contextos de diversidad. }\end{array}$ & Semipresencial & México \\
\hline UNIR & $\begin{array}{l}\text { Máster universitario en Educación Inclusiva e } \\
\text { intercultural }\end{array}$ & Virtual & España \\
\hline Centro de Aprendizaje y superación & Maestría en educación inclusiva & A distancia & México \\
\hline Universidad Baja California & Doctorado en Educación Inclusiva & Semipresencial & México \\
\hline
\end{tabular}

Tabla 3: Programas de posgrado ofertados en el área de educación matemática en Colombia, según nivel de formación posgraduada (Sistema Nacional de Información de la Educación Superior SNIES de Colombia, febrero de 2020).

\begin{tabular}{|l|c|}
\hline Nivel académico y nombre del programa & Cantidad de programas \\
\hline Especialización & 3 \\
\hline Especialización en didáctica matemática física y la educación básica & 2 \\
\hline Especialización en educación matemática & 1 \\
\hline Especialización en pedagogía de la lectoescritura y la matemática & 1 \\
\hline Especialización en necesidades de aprendizaje en lectura, escritura y matemáticas & 3 \\
\hline Maestría & 1 \\
\hline Maestría en didáctica de las matemáticas & 2 \\
\hline Maestría en docencia de la matemática & 7 \\
\hline Maestría en educación énfasis en educación matemáticas y ciencias experimentales & 5 \\
\hline Maestría en educación matemática & \\
\hline Maestría en enseñanza de la matemática & \\
\hline Doctorado & \\
\hline Doctorado en educación matemática & 26 \\
\hline Total posgrados en educación matemática & \\
\hline
\end{tabular}

En el segundo grupo, en Colombia existen nueve programas de posgrado en áreas de inclusión social y/o discapacidad: 4 especializaciones y 5 maestrías; no existe oferta a nivel de doctorado en estas áreas (ver tabla 4). Tres de estos programas de posgrado tienen como área de conocimiento las ciencias de la salud y cinco de ellos en ciencias de la educación; no obstante, ninguno de ellos vincula el área matemática dentro de sus áreas de estudio.

Tabla 4: Programas de posgrado ofertados en el área de inclusión social y/o discapacidad en Colombia, según nivel de formación posgraduada (Datos tomados del SNIES de Colombia, febrero de 2020).

\begin{tabular}{|l|c|}
\hline Nivel académico y nombre del programa & Cantidad de programas \\
\hline Especialización & \\
\hline Especialización en educación y discapacidad & 1 \\
\hline Especialización en discapacidad, educación física, recreación y deporte: adaptados & 1 \\
\hline Especialización en educación: educación especial e inclusión social & 1 \\
\hline Especialización en educación inclusiva & 2 \\
\hline Maestría & 1 \\
\hline Maestría en discapacidad & 1 \\
\hline Maestría en discapacidad e inclusión social & 1 \\
\hline Maestría en psicología de la salud y la discapacidad & 9 \\
\hline Maestría en educación para la inclusión & \\
\hline Total posgrados en el área de inclusión social y discapacidad & \\
\hline
\end{tabular}


En el ámbito regional tampoco existe oferta específica de algún programa de Maestría en Educación Inclusiva. De los programas existentes a nivel nacional en el área de educación matemática (ver tabla 3), en el departamento de Boyacá se ofertan dos maestrías: en Educación Matemática y en Didáctica de la Matemática y dos especializaciones: en Necesidades de Aprendizaje en Lectura, Escritura y Matemáticas y en Didáctica de la Matemática para la Educación Básica; todos ellos ofertados por la Universidad Pedagógica y Tecnológica de Colombia. Mientras tanto, de los programas existentes a nivel nacional en el área de inclusión social y/o discapacidad (ver tabla 4), en este departamento no existe oferta académica a nivel de posgrado. Esta ausencia regional se corrobora también con el cuestionario realizado a los docentes de la provincia centro del departamento de Boyacá, pues el $94 \%$ de ellos indica no conocer opciones de formación profesional en Educación Inclusiva a nivel de posgrado en el departamento.

Dada esta oferta a nivel nacional y regional, las opciones que tienen los docentes para formarse en educación matemática inclusiva es escoger un programa de maestría en matemáticas y profundizar en su trabajo de grado o líneas de investigación respecto a la educación inclusiva, o viceversa, elegir un programa de maestría en educación inclusiva y profundizar durante su carrera en el área matemática. Estas son opciones incompletas para la formación centrada en la educación matemática inclusiva, pues la revisión documental denota la ausencia de un programa con dicho enfoque. Al respecto, se cita a continuación los hallazgos con los expertos.

Los expertos en educación entrevistados indican no conocer algún programa que sea de matemática inclusiva a nivel de posgrado o maestría. Sin embargo, la tabla 5 muestra que algunos de ellos reconocen algunos acercamientos en Colombia respecto a esta temática, por ejemplo, dentro de investigaciones de educación a nivel de pregrado, maestría o doctorado en programas de educación, en las líneas de investigación a nivel de maestría, en la Red Latinoamericana de Matemática Inclusiva y el esfuerzo de algunos programas de Licenciatura en Matemáticas para dotar de herramientas didácticas para la educación inclusiva en los estudiantes.

Tabla 5: Resultados de las entrevistas a expertos respecto al conocimiento de programas de formación en educación matemática inclusiva a nivel de maestría.

\begin{tabular}{|c|c|c|c|}
\hline Experto & $\begin{array}{l}\text { Formación académica y } \\
\text { experiencia del experto }\end{array}$ & Desconocimiento & Acercamientos \\
\hline 1 & $\begin{array}{l}\text { Licenciado en matemáticas y } \\
\text { física. Doctor en Ciencias } \\
\text { Matemáticas. } \\
\text { Investigador Junior (IJ) } \\
\text { Colciencias }\end{array}$ & $\begin{array}{l}\text { "En realidad, no conozco } \\
\text { de ningún programa de } \\
\text { maestría, en Colombia ni } \\
\text { en el exterior, con el } \\
\text { nombre específico de } \\
\text { "maestría en educación } \\
\text { matemática inclusiva" o } \\
\text { con algún nombre } \\
\text { parecido" (P1:3; H. Suárez, } \\
\text { entrevista correo-e, } 8 \text { de } \\
\text { mayo de } 2018 \text { ) }\end{array}$ & $\begin{array}{l}\text { Algunos investigadores están trabajando en } \\
\text { educación matemática inclusiva, pero sus } \\
\text { investigaciones están enmarcadas dentro de } \\
\text { la educación, educación matemática y áreas } \\
\text { afines. Posiblemente dichas investigaciones } \\
\text { las realizan profesores y estudiantes de } \\
\text { maestría o doctorado en educación, } \\
\text { educación matemática o áreas afines" (P1:3; } \\
\text { H. Suárez, entrevista correo-e, } 8 \text { de mayo de } \\
\text { 2018) }\end{array}$ \\
\hline 2 & $\begin{array}{l}\text { Licenciado en Psicología, } \\
\text { Doctor en Ciencias } \\
\text { Pedagógicas (PhD), Doctor en } \\
\text { Ciencias (Dr. Cs.). } \\
\text { Investigador, Profesor y } \\
\text { Consultor Titular. Asesor } \\
\text { Internacional. } \\
\text { Premio Nacional de } \\
\text { Pedagogía de la República de } \\
\text { Cuba. }\end{array}$ & $\begin{array}{l}\text { "Sin embargo no tengo } \\
\text { conocimiento de un } \\
\text { programa de postgrado a } \\
\text { nivel de Maestría sobre } \\
\text { Matemática Educativa } \\
\text { Inclusiva" (P2:2; O. Valera, } \\
\text { entrevista correo-e, } 3 \text { de } \\
\text { mayo de 2018) }\end{array}$ & $\begin{array}{l}\text { "En los últimos años hay un incremento de } \\
\text { Maestrías relacionadas con las Matemáticas, } \\
\text { pero se mueven en dos tendencias } \\
\text { fundamentales: didáctica de las matemáticas } \\
\text { y contenidos matemáticos de mayor } \\
\text { complejidad para su aprendizaje en la } \\
\text { escuela a todos los niveles y el apoyo de las } \\
\text { TIC para su mejora. El enfoque de } \\
\text { educación matemática es menos frecuente y } \\
\text { en el contexto latinoamericano RELME (Red } \\
\text { Latinoamericana de Matemática Educativa) } \\
\text { ha impulsado este tipo de postgrado." (P2:2; } \\
\text { O. Valera, entrevista correo-e, } 3 \text { de mayo de } \\
\text { 2018) }\end{array}$ \\
\hline 3 & $\begin{array}{l}\text { Doctor en psicología. } \\
\text { Profesor de didáctica de las } \\
\text { matemáticas en la Universidad } \\
\text { de Girona (España) y autor de } \\
\text { libros sobre enseñanza y } \\
\text { aprendizaje de las } \\
\text { matemáticas e inclusión. }\end{array}$ & $\begin{array}{l}\text { "no tengo información al } \\
\text { respecto" (P3:4; A. Alsina, } \\
\text { entrevista correo-e, } 23 \text { de } \\
\text { mayo de 2018) }\end{array}$ & \\
\hline
\end{tabular}


Tabla 5: continuación.

\begin{tabular}{|c|c|c|c|}
\hline Experto & $\begin{array}{l}\text { Formación académica y } \\
\text { experiencia del experto }\end{array}$ & Desconocimiento & Acercamientos \\
\hline 4 & $\begin{array}{l}\text { Licenciada en matemáticas y } \\
\text { magíster en docencia e } \\
\text { investigación universitaria. } \\
\text { Docente de la Universidad } \\
\text { Distrital e investigadora en } \\
\text { didáctica de las matemáticas e } \\
\text { inclusión. }\end{array}$ & $\begin{array}{l}\text { "No conozco ninguna" } \\
\text { (P4:5; C. Castro, entrevista } \\
\text { correo-e, } 14 \text { de junio de } \\
2018)\end{array}$ & $\begin{array}{l}\text { Conozco Maestría en Educación con líneas } \\
\text { de investigación como la de la universidad } \\
\text { Distrital (en Bogotá) y la Maestría en } \\
\text { Educación de la Universidad Sergio } \\
\text { Arboleda, que tiene un énfasis en } \\
\text { educación inclusiva y educación para } \\
\text { sordos" (P4:5; C. Castro, entrevista correo- } \\
\text { e, } 14 \text { de junio de 2018) }\end{array}$ \\
\hline 5 & $\begin{array}{l}\text { Licenciada en matemáticas y } \\
\text { doctora en educación. } \\
\text { Docente de la Universidad } \\
\text { Pedagógica Nacional. } \\
\text { Investigador Junior (IJ) } \\
\text { Colciencias } \\
\text { Investigadora en temas de } \\
\text { educación matemática e } \\
\text { inclusión. }\end{array}$ & $\begin{array}{l}\text { "no conozco ningún } \\
\text { programa con esa } \\
\text { especificidad" (P5:2; E. } \\
\text { Torres, entrevista correo-e, } \\
14 \text { de agosto de 2018). }\end{array}$ & $\begin{array}{l}\text { "... algunos programas de pregrado a nivel } \\
\text { local vienen haciendo esfuerzos desde su } \\
\text { currículo para formar a los futuros } \\
\text { licenciados en matemáticas con algunas } \\
\text { herramientas que permitan atender a las } \\
\text { poblaciones que son consideradas } \\
\text { especiales o vulnerables y se incluyen al } \\
\text { aula regular, tal es el caso del programa de } \\
\text { la licenciatura en educación básica con } \\
\text { énfasis en matemáticas (hoy licenciatura en } \\
\text { matemáticas de la Universidad Distrital)" } \\
\text { (P5:2; E. Torres, entrevista correo-e, } 14 \text { de } \\
\text { agosto de 2018). }\end{array}$ \\
\hline
\end{tabular}

Se observa entonces que la vinculación entre la educación inclusiva y el área matemática se ha adelantado dentro de programas de formación en educación ya establecidos, ya sea como iniciativa personal de profesores o estudiantes de profundizar en dicha temática o como enfoques específicos de algunos de estos programas. Estos acercamientos no constituyen oferta educativa puntual respecto a la educación matemática inclusiva, pero evidencia las posibilidades existentes respecto al diseño de un programa a nivel de maestría que supla esta necesidad.

En la indagación documental también se encontraron algunos avances respecto a la educación inclusiva y la educación matemática inclusiva, pero ninguna de estas iniciativas se ha materializado en la oferta de formación profesional posgraduada a nivel de maestría. Por ejemplo, a nivel internacional, se han desarrollado sistemas que aumentan la accesibilidad interactiva del contenido matemático EPUB3 para estudiantes con discapacidad visual (Brzostek et al., 2019), se ha planteado el ajedrez en el proceso de aprendizaje de matemáticas con posibilidad de ser adaptado de manera inclusiva (Dvoryatkina et al., 2019), se han implementado aplicaciones móviles para enseñar operaciones básicas a estudiantes con Síndrome de Down (Tangarife, 2018), y se han utilizado lectores de pantalla a fin de fortalecer el aprendizaje de personas no videntes (Basantes et al., 2018).

A nivel regional, se han propuesto referentes curriculares para la formación de profesores en y para la diversidad en las áreas de matemáticas, lenguaje y comunicación y ciencias naturales, considerando la incorporación de tecnologías de la información (ALTER-NATIVA, 2013); y por otro, en Colombia se han realizado algunas intervenciones prácticas desde la matemática inclusiva en la formación de futuros licenciados y algunas propuestas de talleres complementarios de formación en esta misma área para estudiantes (Castro y Torres, 2017). Recientemente se ha propuesto también la enseñanza de algebra desde las tecnologías de la información y las comunicaciones dirigido a estudiantes con discapacidad auditiva (Lancheros et al., 2018).

También la Gobernación de Boyacá ha realizado en los últimos años convenios de soporte profesional con entidades educativas como la Universidad Pedagógica y Tecnológica de Colombia o con la Fundación Centros de Aprendizaje Neuroharte para el apoyo a la inclusión educativa de la población con discapacidad. De igual manera, se han planteado foros en los que se han involucrado instituciones como la Escuela Taller de Boyacá o premiaciones de docentes que hayan tenido experiencias exitosas en la atención de este tipo de población. Sin embargo, estos conjuntos de acciones se basan en consultorías de expertos externos a la institución educativa y no en el fortalecimiento profesional de los docentes.

En conjunto, la evidencia documental y oral muestran la ausencia de un programa de formación a nivel de maestría en educación matemática inclusiva a nivel internacional y en Colombia. No obstante, existen algunos avances investigativos y acercamientos académicos al respecto que pueden consolidarse en la creación de dicho programa y así profesionalizar a los docentes de matemáticas del departamento de Boyacá. 


\section{Demanda del programa en los docentes de matemáticas}

En el departamento de Boyacá hay 754 profesores del área de matemáticas para el año 2018 y el 19.5\% se encuentran en la provincia centro con 147 de ellos, siendo la mayor participación entre todas las subregiones (Secretaría de Educación de Boyacá, 2018). Entre los docentes de la provincia centro, sólo el 17.7\% posee educación a nivel de maestría, mientras que el $73.3 \%$ restante tienen título profesional, licenciatura y especialización. Por tanto, si la motivación de los docentes a especializarse a nivel de maestría es subir el escalafón y mejorar los ingresos, existe un $73.3 \%$ de los docentes que pueden profesionalizarse en educación matemática inclusiva. Por otro lado, la tabla 6 evidencia los principales resultados del cuestionario aplicado a docentes y resume los hallazgos respecto a las cuatro categorías y variable definidas para la demanda.

Tabla 6: Resultados de cuestionarios a profesores de matemáticas de la provincia Centro del departamento de Boyacá.

\begin{tabular}{|c|c|c|}
\hline Categoría & Variable & Participación relativa obtenida \\
\hline \multirow{2}{*}{$\begin{array}{l}\text { Realización de estudios } \\
\text { respecto a la educación } \\
\text { inclusiva }\end{array}$} & Posee formación en educación inclusiva & $\begin{array}{l}\text { Si } 6 \% \\
\text { No } 94 \%\end{array}$ \\
\hline & Posee formación en educación matemática inclusiva & $\begin{array}{l}\text { Si } 0 \% \\
\text { No } 100 \%\end{array}$ \\
\hline \multirow{4}{*}{$\begin{array}{l}\text { Autovaloración de la } \\
\text { capacidad de atención } \\
\text { de la diversidad }\end{array}$} & $\begin{array}{l}\text { Sentimiento de preparación en la atención de } \\
\text { estudiantes en condición de vulnerabilidad, discapacidad } \\
\text { y capacidades y talentos excepcionales }\end{array}$ & $\begin{array}{l}\text { Muy preparado } 5 \% \\
\text { Moderadamente preparado } \\
16 \% \\
\text { En muchos casos no muy } \\
\text { preparado } 39 \% \\
\text { Para nada preparado } 40 \%\end{array}$ \\
\hline & $\begin{array}{l}\text { Presencia de inconvenientes a la hora de enseñar y } \\
\text { atender estudiantes en condición de discapacidad, } \\
\text { vulnerabilidad y capacidades y talentos excepcionales }\end{array}$ & $\begin{array}{l}\text { Si } 85 \% \\
\text { No } 15 \%\end{array}$ \\
\hline & Capacidad para dar solución a dichos inconvenientes & $\begin{array}{l}\text { Si } 70 \% \\
\text { No } 30 \%\end{array}$ \\
\hline & $\begin{array}{l}\text { Presencia de estrategias de atención a población con } \\
\text { discapacidad, vulnerabilidad y capacidades y talentos } \\
\text { excepcionales en la Institución Educativa }\end{array}$ & $\begin{array}{l}\text { Si } 83 \% \\
\text { No } 17 \%\end{array}$ \\
\hline $\begin{array}{l}\text { Conocimiento de } \\
\text { programas académicos } \\
\text { sobre educación } \\
\text { inclusiva en posgrado }\end{array}$ & $\begin{array}{l}\text { Conocimiento de opciones de formación profesional en } \\
\text { Educación Inclusiva a nivel de posgrado en el } \\
\text { departamento de Boyacá }\end{array}$ & $\begin{array}{l}\text { Si } 6 \% \\
\text { No } 94 \%\end{array}$ \\
\hline \multirow{2}{*}{$\begin{array}{l}\text { Interés por tomar } \\
\text { estudios de posgrado a } \\
\text { nivel de maestría }\end{array}$} & $\begin{array}{l}\text { Preferencia para tomar como opción de formación } \\
\text { profesional una Maestría en Educación Matemática } \\
\text { Inclusiva }\end{array}$ & $\begin{array}{l}\text { Si } 91 \% \\
\text { No } 9 \%\end{array}$ \\
\hline & $\begin{array}{l}\text { Modalidad en que tomaría la Maestría en Educación } \\
\text { Matemática Inclusiva }\end{array}$ & $\begin{array}{l}\text { Presencial } 15 \% \\
\text { Semipresencial } 20 \% \\
\text { Virtual } 65 \%\end{array}$ \\
\hline
\end{tabular}

Se observa que pocos docentes de la provincia Centro del departamento de Boyacá ellos poseen formación profesional en educación inclusiva o matemática inclusiva. Esta necesidad está acompañada por el sentimiento de poca preparación para atender estudiantes en condición de vulnerabilidad, discapacidad y capacidades y talentos excepcionales y las consecuentes dificultades a la hora de enseñar y atender estudiantes con diversidad. En consecuencia, la mayoría de los docentes encuestado tomaría como opción de formación profesional una Maestría en Educación Matemática Inclusiva. En conjunto, se observa que no solo existe un mercado potencial para el programa de formación debido a la existencia de pocos docentes con nivel de maestría y las dificultades presentadas al atender población con discapacidad y diversidad, sino que existe la disposición para optar por la Maestría en Educación Inclusiva.

\section{Oportunidades de desempeño para los egresados del programa}

Existen oportunidades de desempeño en el sector público para docentes profesionales en matemática inclusiva. Por un lado, el decreto 1421 de 2017 plantea la necesidad y obligatoriedad de tener docentes de apoyo pedagógico en las instituciones educativas, cargo en el cual los docentes tienen como función principal acompañar pedagógicamente a los docentes de aula que atienden estudiantes con discapacidad. Esta es un área de desempeño para el egresado del programa de Maestría en Educación Matemática Inclusiva. 
Por otro lado, el plan de desarrollo departamental 2016 - 2019 tiene como subprograma inclusión de población con necesidades educativas especiales y población vulnerable, cuyo desarrollo requiere del apoyo de profesionales en educación inclusiva y matemática inclusiva. Dentro de sus metas se destaca el desarrollo de proyectos pedagógicos en instituciones educativas con población indígena, negra tradicional, género y con necesidades educativas (Gobernación de Boyacá, 2016). También se identifican programas para inclusión social y espacios de deporte para personas con discapacidad y diferentes grupos vulnerables. Esto implica poseer docentes y personal calificado para atender dichas poblaciones y una oportunidad ocupacional para los egresados del programa de maestría en mención.

En otros ámbitos, el Magíster en Educación Matemática Inclusiva puede desempeñarse en investigación académica tanto en universidades públicas como privadas o instituciones del orden nacional adscritas al Departamento Administrativo de Ciencia, Tecnología e Innovación (COLCIENCIAS). De igual manera, podrá desempeñar labores de formulación de políticas públicas del departamento, relacionadas con población con discapacidad y población vulnerable. En el sector privado existen posibilidades de desempeño en instituciones educativas existentes que quieran empezar procesos de inclusión social. En este sentido, si el maestrante ya ha desarrollado capacidad de emprendimiento puede emprender instituciones educativas con enfoque de inclusión, puede generar proyectos productivo-educativos en la sociedad en que se encuentra, o puede generar empresas contratistas que apoyen procesos del gobierno departamental y nacional.

Estos roles profesionales se confirman en los campos ocupacionales recomendado por los expertos para el egresado del programa de maestría en Educación Matemática Inclusiva (Ver tabla 7), las cuales se centran en la docencia, la investigación, la asesoría y consultoría y la gestión educativa; todos ellos apalancados en los conocimientos de la enseñanza inclusiva de la matemática. Se observa entonces que existen oportunidades de desempeño en el sector educativo debido a la promoción de la educación inclusiva en el país, en el sector público gracias a los planes departamentales de inversión o las prioridades de investigación o en el sector privado en instituciones educativas o emprendimientos propios.

Tabla 7: Resultados de las entrevistas a expertos respecto al campo ocupacional en el que se puede desempeñar el egresado del programa.

\begin{tabular}{|c|l|}
\hline Experto & \multicolumn{1}{c|}{ Campos ocupacionales propuestos } \\
\hline 1 & $\begin{array}{l}\text { Profesor de matemáticas en los diferentes niveles de la educación. } \\
\text { Docente administrativo en instituciones de educación, en diferentes niveles. } \\
\text { Investigador en el área de la educación matemática inclusiva. } \\
\text { Gestor y ejecutor de proyectos de capacitación y cualificación de profesores, en educación matemática } \\
\text { inclusiva. } \\
\text { Asesor en educación matemática inclusiva y orientador de comunidades educativas que se relacionan con } \\
\text { educación matemática inclusiva. }\end{array}$ \\
\hline 2 & $\begin{array}{l}\text { Docencia } \\
\text { Gestor de modelos educativos y diseños curriculares } \\
\text { Investigador en contenidos didácticos matemáticos y creación de experiencias de innovación educativa } \\
\text { matemática. }\end{array}$ \\
\hline 3 & $\begin{array}{l}\text { Docencia con enfoque en diversidad. } \\
\text { Investigador }\end{array}$ \\
\hline 4 & $\begin{array}{l}\text { Profesional con capacidades para reconocer y trabajar con la diversidad. } \\
\text { Planeación y gestión institucional respecto a rutas didácticas, aplicación del DUA y política pública. } \\
\text { Gestor de aula para realizar las adaptaciones necesarias para la enseñanza matemática. } \\
\text { Evaluador, teniendo en cuenta la flexibilización curricular necesaria. } \\
\text { Investigador en su propia práctica y tenciones precisas en el aula inclusiva. }\end{array}$ \\
\hline
\end{tabular}

\section{DISCUSIÓN}

Los hallazgos con expertos y revisión documental demuestran que existen diversos acercamientos a nivel de posgrado que vinculan la educación inclusiva y el área matemática, pero no se ha concretado en un programa de maestría cuyo enfoque sea exclusivamente ese. Estas dos áreas tienen suficientes puntos de encuentro para diseñar un pensum académico en este nivel post gradual y las encuestas con los docentes demuestran la necesidad de este tipo de formación y la disposición a tomarla. También las fuentes de información mencionadas comprueban que existe necesidad y disposición de los docentes por este tipo de formación, además de diversas oportunidades que se vislumbran para el desempeño ocupacional de quienes se formen en la educación matemática inclusiva. 
Estos resultados posibilitan para indagaciones posteriores la determinación de la factibilidad y el diseño curricular el programa de maestría. En el primer caso, implica determinar el entorno legal para la oferta del programa y las capacidades financieras, institucionales, tecnológicas y humanas para ofertarlo. En el segundo caso, abarca la fundamentación pedagógica, epistemológica, profesional, socioeconómica y político-legal del programa, junto con la determinación del perfil profesional, organización del currículo, estrategias didácticas y sistemas de evaluación y acreditación del título.

De esta manera, los resultados de esta investigación contribuyen al diseño curricular del programa de maestría en Educación Matemática Inclusiva, inicialmente en Boyacá y posteriormente en el país, lo cual evita a mediano y largo plazo la problemática expuesta por Lewin (2014): la exclusión o perjuicio de estudiantes de grupos minoritarios. Específicamente, las necesidades de formación y las oportunidades de desempeño identificadas en esta investigación son la base para estructurar el perfil profesional y las competencias del programa de maestría dentro del proceso de diseño curricular. Mientras tanto, el estudio de la oferta permite validar la oportunidad de diseñar dicho programa e identificar los avances en el área (Castro y Torres, 2017) que, aunque no se han materializado en un programa de formación posgraduada, favorecen la fundamentación pedagógica y profesional de la Maestría en Educación Matemática Inclusiva.

La baja preparación de los docentes para educar en la discapacidad y la diversidad evidencia la baja capacidad estatal para formar a los docentes en estas áreas (ALTER-NATIVA, 2013; Faragher \& Clarke, 2016; Hurtado \& Agudelo, 2014), lo que conduce a que se continúen con dificultades metodológicas, pedagógicas y curriculares para la enseñanza y aprendizaje de las matemáticas con grupos diversos (Lambert \& Tan, 2017; Obando et al., 2020). Con los hallazgos de este estudio se refuerza la idea de la importancia de avanzar hacia oportunidades más inclusivas para la enseñanza y aprendizaje de las matemáticas. Pese algunos avances individuales al respecto (Basantes et al., 2018; Brzostek et al., 2019; Dvoryatkina et al., 2019; Tangarife, 2018), aún faltan diversos caminos por recorrer en la investigación, la formación de docentes y la extensión de la cultura inclusiva en las clases de matemáticas.

\section{CONCLUSIONES}

De acuerdo a los resultados de este estudio y su discusión se puede concluir de manera general que existen necesidades de formación en educación matemática inclusiva en los docentes departamento de Boyacá y poca oferta educativa que las supla, lo cual representa una oportunidad para diseñar un programa de maestría para esta región. Específicamente se extraen las siguientes tres conclusiones: 1) En el ámbito internacional, colombiano y del departamento de Boyacá no existe oferta de programas de posgrado cuyo enfoque sea la Educación Matemática Inclusiva; aun así, se reconocen iniciativas de investigación que pueden fundamentar el diseño curricular un programa de maestría en esta área; 2) Los docentes de matemáticas de Boyacá tienen poca profesionalización en educación inclusiva, reconocen las dificultades que poseen para enseñar en contextos de discapacidad y diversidad y demuestran disposición de formarse en educación matemática inclusiva a nivel de maestría; 3) Existen oportunidades de desempeño para el magíster en educación matemática inclusiva en instituciones públicas y privadas.

\section{REFERENCIAS}

Acebedo, M. J., Aznar, I., y Hinojo, F. J., Instrumentos para la Evaluación del Aprendizaje Basado en Competencias: Estudio de caso, doi: 10.4067/S0718-07642017000300012, Información Tecnológica, 28(3), 107-118, (2017).

Ainscow, M. Diversity and Equity: A Global Education Challenge, doi: 10.1007/s40841-016-0056-x, New Zealand Journal of Educational Studies, 51(2), 143-155. (2016)

Ainscow, M., y Miles, S.. Making Education for all Inclusive: Where Next?, doi: 10.1007/s11125-008-9055-0, Prospects: Comparative Journal of Curriculum, Learning and Assessment, 38(145), 15-34, (2008)

Alsina, Á., y Planas, N., Matemática inclusiva: propuestas para una educación matemática accesible. Narcea de ediciones, Madrid, España, (2010)

ALTER-NATIVA, Referentes curriculares con incorporación de tecnologias para la formación del profesorado de matemáticas en y para la diversidad. Universidad Distrital Francisco José de Caldas, Bogotá D.C., Colombia, (2013)

Baglieri, S., Valle, J. W., Connor, D. J., y Gallagher, D. J., Disability Studies in Education: The Need for a Plurality of Perspectives on Disability, doi: 10.1177/0741932510362200, Remedial and Special Education, 32(4), 267-278, (2011)

Basantes, A. V., Guerra, F. E. y otros dos autores, Los Lectores de Pantalla: Herramientas Tecnológicas para la Inclusión Educativa de Personas no Videntes. Doi: 10.4067/S0718-07642018000500081, Información tecnológica, 29(5), 81-90, (2018).

Brzostek, J., Rubin, M., y Salamończyk, A, Enhancement of Math Content Accessibility in EPUB3 Educational Publications, doi: 10.1080/13614568.2019.1664645, New Review of Hypermedia and Multimedia, 25(1-2), 31-56, (2019). 
Castro, C., y Torres, E., La Educación Matemática Inclusiva: Una Experiencia en la Formación de Estudiantes para Profesor. Infancias Imágenes, 16(2), 295-304, (2017)

Dvoryatkina, S. N. y otros cuatro autores, Synergetic Effects Manifestation by Founding Complexes Deployment of Mathematical Tasks on the Chessboard. doi: 10.33225/pec/19.77.08, Problems of Education in the 21st Century, 77(1), 821, (2019)

Faragher, R., y Clarke, B., Teacher Identified Professional Learning Needs to Effectively Include a Child With Down Syndrome in Primary Mathematics: Professional Learning Needs for Primary Mathematics, doi: 10.1111/jppi.12159, Journal of Policy and Practice in Intellectual Disabilities, 13(2), 132-141, (2016)

Florian, L., y Camedda, D., Enhancing Teacher Education for Inclusion, doi: 10.1080/02619768.2020.1707579, European Journal of Teacher Education, 43(1), 4-8, (2020).

Gobernación de Boyacá, Plan Departamental de Desarrollo de Boyacá “Creemos en Boyacá tierra de paz y libertad” 2016 - 2019. Secretaría de Planeación Departamental, Tunja, Colombia, (2016)

Hurtado, T., y Agudelo, M., Inclusión Educativa de las Personas con Discapacidad en Colombia. Revista CES Movimiento y Salud, ISSN: 2357-562X, 2, 45-55, (2014).

Messiou, K. y otros ocho autores, Learning From Differences: A Strategy for Teacher Development in Respect to Student Diversity, doi: 10.1080/09243453.2014.966726, School Effectiveness and School Improvement, 27(1), 45-61, (2016).

Lancheros, D. J., Bohorquez, L., Cortes, L., y Villarraga, M., Algebra Teaching: An Inclusive Experience from the Information Technologies and Communications, doi: 10.23919/CISTI.2018.8399333, 2018 13th Iberian Conference on Information Systems and Technologies (CISTI), 1-6, (2018)

Lambert, R., y Tan, P., Conceptualizations of Students with and without Disabilities as Mathematical Problem Solvers in Educational Research: A Critical Review, doi: 10.3390/educsci7020051, Education Sciences, 7(2), 51, (2017)

León, A. P., Risco del Valle, E., y Alarcón, C., Estrategias de Aprendizaje en Educación Superior en un Modelo Curricular por Competencias, doi: 10.1016/j.resu.2015.03.012, Revista de la Educación Superior, 43(172), 123-144, (2014)

Lewin, D. What's the Use of Ethical Philosophy? The Role of Ethical Theory in Special Educational Needs, doi: 10.1080/08856257.2014.933549, European Journal of Special Needs Education, 29(4), 536-547, (2014)

Matsuura, K., Preface. Inclusive Education. Prospects: Comparative Journal of Curriculum, Learning and Assessment, 38(145), 1-4, (2008)

Moscoso, J. N., Los Métodos Mixtos en la Investigación en Educación: Hacia un Uso Reflexivo, doi: 10.1590/198053143763, Cadernos de Pesquisa, 47(164), 632-649, (2017)

Obando, G., Pontón, T., Parada, S.-E., y Villa, J. A., Research into Cognition and Numerical Thinking in Colombia, doi: 10.1080/02109395.2020.1748841, Studies in Psychology, 41(2), 319-347, (2020)

Petreñas, C., Puigdellívol, I., y Jardí, A. Do Educational Support Policies Always Favour Overcoming Inequalities? The Situation in Spain, doi: 10.1080/09687599.2020.1788510 Disability y Society, O(0), 1-24, (2020).

Santiváñez, V. Diseño curricular a partir de competencias. Ediciones de la U, Bogotá D.C., Colombia, (2013)

Shogren, K. A., y Wehmeyer, M. L., Using the Core Concepts Framework to Understand Three Generations of Inclusive Practices, doi: 10.1352/2326-6988-2.3.237, Inclusion, 2(3), 237-247, (2014).

Secretaría de Educación de Boyacá, Respuesta Derecho de Petición, Tunja, Colombia, (2018).

Sistema Integrado de Matricula (SIMAT)- Secretaría de Educación de Boyacá, Consolidado de matrícula oficial regular. Secretaría de Educación de Boyacá, Tunja, Colombia, (2019).

Tangarife, D. La Enseñanza de las Matemáticas a Personas con Síndrome de DOWN Utilizando Dispositivos Móviles, doi: 10.24320/redie.2018.20.4.1751, Revista Electrónica de investigación Educativa, 20(4), 144-153, (2018).

Tan, P., Lambert, R., Padilla, A., y Wieman, R., A Disability Studies in Mathematics Education Review of Intellectual Disabilities: Directions for Future Inquiry and Practice, doi: 10.1016/j.jmathb.2018.09.001, The Journal of Mathematical Behavior, 54, 100672, (2019)

Tan, P., y Padilla, A., Prospective Mathematics Teachers' Engagement with Inclusive Equity: An Exploratory Case Study in Anti-ableist Sociopolitical Grounding, doi: 10.1016/j.tate.2019.06.007, Teaching and Teacher Education, 86, 102853, (2019) 
